\title{
Sentidos sobre el cuidado en salud mental: una revisión bibliográfica
}

\author{
Senses about mental health care: a literature review
}

Renata Guerda de Araújo Santos', Lupicinio Íñiguez-Rueda1

DOI: $10.1590 / 0103-1104202112818$

RESUMEN Este texto presenta una revisión bibliográfica realizada en la Biblioteca Virtual de Salud/ Psicología (BVS-PSI/Brasil) con el objetivo de comprender los sentidos del cuidado en la atención psicosocial a partir de los artículos científicos brasileños disponibles. Realizamos una búsqueda con los descriptores del DeCS/MeSH para acceder a las publicaciones disponibles. Hicimos una revisión de esta literatura, describimos el contenido de los artículos, identificamos los repertorios lingüísticos presentes y problematizamos los diversos sentidos del cuidado en la perspectiva de la atención psicosocial. Trabajamos con las contribuciones de la Psicología Discursiva y del movimiento construccionista para comprender la complejidad y la polifonía de ese campo. Los resultados se han agrupado en tres categorías: La Política Pública y la Reforma Psiquiátrica en Brasil; Modelos de asistencia y las controversias en la gestión y organización del proceso de trabajo; y la Salud Colectiva. Podemos visualizar, de manera amplia y dinámica los tránsitos conceptuales, a veces desconectados y reduccionistas, a veces como una vía importante para la transformación de las prácticas de salud mental en la atención psicosocial.

PALABRAS-CLAVE Salud mental. Servicios de salud mental. Modelos de atención de salud.

\begin{abstract}
This text presents a bibliographic review carried out at the Virtual Library in Health/Psychology/ Brazil (BVS-PSI/Brazil) with the objective of understanding the meanings of care in psychosocial care based on available Brazilian scientific articles. We conducted a search with the DeCS/Mesh descriptors to access the quantity of available publications. We did a bibliographic review of this literature, described the content of the articles, identified the linguistic repertories present and problematized problematized the various meanings of care in the perspective of psychosocial care. We worked with the contributions of Discursive Psychology and the constructionist movement to understand the complexity and polyphony of that field. The results have been grouped into three categories: Public Policy and Psychiatric Reform in Brazil; Models of assistance and controversies in the management and organization of the work process; and Collective Health. We can visua-lize, in a broad and dynamic way, the conceptual, sometimes disconnected and reductionist transits, sometimes as an important way for the transformation of mental health practices into psychosocial care.
\end{abstract}

KEYWORDS Mental health. Mental health services. Healthcare models. 


\section{Introducción}

La Atención Psicosocial en Brasil está marcada por una ruptura paradigmática engendrada por transformaciones en el objeto históricamente delimitado por la psiquiatría ${ }^{\mathbf{1}}$. La influencia de la psiquiatría de sector francés, de la psiquiatría comunitaria americana, de la antipsiquiatría, de la psicoterapia institucional y de la psiquiatría italiana, van imprimiendo bifurcaciones en el campo de la salud mental, y produciendo efectos en el modo de producción del cuidado.

En la metamorfosis de la naturaleza de la institución psiquiátrica, diversos cambios fueron operados buscando restablecer un potencial terapéutico a las prácticas adoptadas a lo largo de los años ${ }^{2}$. Estos movimientos se caracterizaban por acciones que buscaban calificarla sin necesariamente cambiar sus bases científicas y sus aspectos filosóficos, metodológicos, ontológicos y epistemológicos.

En el contexto brasileño, la contribución de estas perspectivas, sumada al momento histórico de la lucha por la redemocradización del país (1964-1985), ha producido un efecto de transformación del proyecto clínico de la psiquiatría y su modelo manicomial ${ }^{3}$. Se trata de una construcción política de cuestionamiento de la resolutividad de la atención en salud mental y sus procedimientos higienistas de control: camisa de fuerza, electroshock, uso abusivo de medicamentos, aislamiento, malos tratos, etc.

Este movimiento se fortalece a partir de la movilización protagonizada por la Reforma Sanitaria iniciada a finales de la década de 1970. A partir de ella, las intervenciones en salud mental buscaron promover nuevas posibilidades de calificar las condiciones y modos de vida, guiándose por la producción de salud y no restringiéndose a la cura de enfermedades 4 .

Así, la salud mental se orienta a través de las prácticas de la integralidad y se espera una mayor capacidad para comprender las necesidades de las personas con respuestas adecuadas a sus demandas 5 . La integralidad es uno de los principios doctrinales de la política de salud y tiene por objetivo conjugar las acciones dirigidas a la materialización de la salud como un derecho ${ }^{6}$.

La política de salud mental se ha constituido desde dos marcadores legales importantes: la Ley $10.216^{7}$, que dispone sobre la protección y los derechos de las personas portadoras de trastornos mentales y redirecciona el modelo asistencial de acuerdo con lo establecido en la Ley Orgánica del Sistema Único de Salud (SUS), y la Red de Atención Psicosocial (Raps), cuya finalidad es la creación, ampliación y articulación de puntos de atención a la salud para personas con sufrimiento, trastorno mental o con necesidades derivadas del uso de crack, alcohol y otras drogas.

La Raps fue creada en 2011 como estrategia para la ordenación de la atención de la salud mental en el SUS. Está compuesta por varios establecimientos públicos, a los cuales se les asigna diferentes responsabilidades para la integración y resolutividad. La red incluye servicios vinculados a sus variados componentes de cuidado: atención básica, atención psicosocial especializada, emergencia, vivienda transitoria, hospitalaria y estrategias de desinstitucionalización y rehabilitación ${ }^{8}$.

El redireccionamiento del modelo psiquiátrico asilar hacia la producción de un cuidado en redes, moviliza dimensiones teórico-conceptuales, técnico-asistenciales, jurídico-políticas y socioculturales ${ }^{2}$. Es decir, las prácticas de cuidado deben ser longitudinales y coordinadas de forma continua en la vida de las personas y de las poblaciones. Reconocido como atributo esencial para los modelos de atención amplios y efectivos, el cuidado pasa a ser dispositivo estratégico en la conducción de políticas, programas y estrategias de salud en Brasil9.

La elección del término cuidado para hablar de prácticas de atención y asistencia en salud mental podría sugerir una coherencia definida de antemano o una categoría general. Sin embargo, se trata de un término atravesado por diversas tensiones, entre ellas, el modo 
prescriptivo queja-conducta, ya que las necesidades en salud mental tienen como respuesta estándar la derivación a consulta médica o al nivel especializado. Hay también iniciativas que buscan componer estrategias conectadas e intersectoriales con las necesidades de la población, además de las lógicas que sostienen y dirigen el cuidado a partir de la conducción en la Raps.

Muchas de esas prácticas se desarrollan en el acto de cuidar, teniendo en cuenta las dimensiones subjetivas, relacionales y singulares. Sin embargo, presuponemos que, además de la interacción interpersonal, la producción del cuidado en salud mental exige la construcción de herramientas que produzcan vínculos, requiere la responsabilización colectiva de los profesionales y un sistema de salud con organizaciones orientadas por políticas estructurantes y convergentes.

No obstante, las conquistas en el campo de las políticas públicas de salud mental a lo largo de los años están siendo amenazadas por la falta de inversión en el SUS, cronificada por el actual gobierno 2019/2022 ${ }^{10}$ y su política neoliberal. Basada en la austeridad, privatización y desregulación, tal política se acentúa por la grave crisis económica e ideológica que ataca los principios constitucionales en lo que se refiere a la atención salud: universalidad, integralidad, equidad y participación social.

Así, lo que está configurado como una importante expansión del acceso a la atención primaria, a la atención de emergencia, a las inmunizaciones (vacunas), a la atención prenatal, a los servicios especializados y a los servicios hospitalarios, ha aparecido también como el principal problema en el país ${ }^{9}$. Tal vez esta paradoja pueda explicarse por el hecho de que la aplicación del SUS sigue siendo parcial, de forma que es necesario garantizar la sostenibilidad del sistema con el fin de continuar con la reforma del Estado y el modelo de gestión destinado a corregir varios obstáculos estructurales y funcionales.

En este sentido, hace falta encontrar un diseño organizacional y reconstruir el marco institucional del SUS para mejorar su carácter público, así como reconocer las disputas que surgen en este escenario: institucional, sistemas de gestión, financiación, planificación y evaluación de impacto para un proyecto de salud en defensa de la vida.

Por lo tanto, presentamos los resultados de una revisión realizada en la Biblioteca Virtual de Salud/Psicología (BVS-PSI/Brasil) con el objetivo de comprender los sentidos del cuidado en la atención psicosocial presentes en los artículos científicos brasileños disponibles. Y como objetivos específicos: identificar los repertorios linguísticos utilizados para definir el cuidado en la atención psicosocial, y problematizar los límites inexactos entre el conocimiento y las prácticas de producción del cuidado en el campo de la atención psicosocial.

\section{Metodología}

Desde el momento que elegimos un tema de investigación nos insertamos instantáneamente en el campo-tema, el cual se configura en las situaciones que ocurren en la cotidianeidad, a través de conversaciones, flujos, encuentros que pasan a formar parte del proceso de investigación"1. Trabajamos aquí con las contribuciones teóricas y metodológicas de la psicología discursiva y del movimiento construccionista. En este sentido, comprendemos la ciencia como una práctica social, producto de las interacciones humanas históricamente situadas ${ }^{\mathbf{1 2}}$.

Las diversas versiones y explicaciones sobre las cosas del mundo son protagonizadas en actos creativos, es decir, en composiciones polifónicas entre investigadores/as, comunidades científicas, estableciendo campos de diálogos y negociaciones, componiendo una red compleja y heterogénea de producción de realidades. La heterogeneidad de las partes será siempre considerada antes de la unidad del todo, y las totalidades se consideran efectos provisionales, transitorios e inacabados ${ }^{13}$. O sea, para este análisis, comprendemos que el lenguaje se conecta y se construye con propósitos y para obtener consecuencias determinadas. Así, 
interesa la variabilidad de sus usos, tanto como un índice de la función como un índice de las distintas maneras en que se puede fabricar una explicación ${ }^{\mathbf{1 4}}$.

Este trabajo busca acercarse a la crítica a las ciencias modernas y de alguna forma posicionarse superando las dicotomías: realismo y construccionismo; sociedad e individuo; sujeto y objeto; teoría y práctica; cuantitativo y cualitativo; la ciencia y la vida cotidiana. En definitiva, posicionarse desde una crítica continua a la política y a los métodos del positivismo ${ }^{15}$.

\section{Procedimientos}

La investigación bibliográfica implica un conjunto ordenado de procedimientos de búsqueda no aleatorios ${ }^{\mathbf{1 6}}$, que posibilita la exploración de los modos por los que determinados temas se hacen relevantes para la ciencia, cuáles medios se organizan y sus condiciones de producción del conocimiento, con el fin de discutirlas críticamente ${ }^{17}$. Para esta en específico, hemos adoptado la búsqueda en bases de datos, como una estratégia central de las investigación bibliográfica, considerando que esta modalidad consiste en el levantamiento, selección, organización y clasificación de la información relacionándola con los objetivos planteados.

Elegimos hacer la investigación en la BVS-PSI/Brasil por ser un portal de la máxima relevancia en el contexto de la salud colectiva y de la salud pública en el país, que son precisamente los más importantes campos de aproximación a la atención psicosocial. La BVS-PSI/ Brasil es una iniciativa del Conselho Federal de Psicologia en asociación con la Universidade de São Paulo, la Organización Mundial de la Salud, la Organización Panamericana de la
Salud y el Bireme (Centro Latinoamericano y del Caribe de Información en Salud), siendo posible tener acceso al portal porque utiliza el sistema de publicación del SciELO y edita más de 52 títulos de revistas de los países de América Latina y el Caribe.

Se trata de un levantamiento realizado a partir de fuentes secundarias, entre los meses de noviembre y diciembre de 2018 y enero de 2019. Las primeras entradas se hicieron para reconocer los descriptores disponibles en los Descriptores en Ciencias de la Salud (DeCS) para luego hacer una elección que pudiera incorporar los objetivos de la investigación.

El DeCS es un vocabulario estructurado y trilingüe creado por el Centro LatinoAmericano e do Caribe de Informação em Ciências da Saúde. El DeCS sirve como un lenguaje único en la indexación de artículos de textos científicos para que para que sea utilizado en la investigación y recuperación de asuntos de la literatura científica en las fuentes de información disponibles en la BVS.

Considerando que el objetivo de esta búsqueda es producir también estrategias de visibilidad sobre las publicaciones dentro del campo de la atención psicosocial, las búsquedas se realizaron utilizando el operador booleano $A N D$ para facilitar el acceso a las informaciones deseadas.

Los criterios de elección de los descriptores buscaron contemplar la complejidad del campo de investigación, considerando que los modelos de atención psicosocial no son uniformes y cada uno de ellos guarda una especificidad local-regional, histórica, cultural, y económica. Para acceder a las publicaciones, trabajamos con el uso de dos marcadores que han posibilitado visualizar la heterogeneidad de este campo, tal como en el cuadrol: 
Cuadro 1. Marcadores para la búsqueda

\begin{tabular}{lll}
\hline & MARCADORES CENTRALES & MARCADORES PERIFÉRICOS \\
\cline { 2 - 3 } DESCRIPTORES & "Saúde Mental" & "Serviços de Saúde" \\
& "Assistência Integral à Saúde" & "Serviços de Saúde Mental" \\
& "Padrão de Cuidado" & "Serviços Comunitários de Saúde Mental" \\
\hline
\end{tabular}

Fuente: Elaboración propia.

Los criterios para la elección de estos marcadores partieron de la definición de campo como una matriz ${ }^{18}$ compuesta de saberes y prácticas de otras categorías y actores sociales, que por sus movimientos y transformaciones son capaces de contribuir con los cambios del modelo de atención y de la lógica con que funcionan los servicios de salud en general. Para analizar los sentidos del cuidado presentes en estas publicaciones, empezamos las búsquedas por el campo (Marcadores Centrales) y seguidamente elegimos descriptores que pudieran hacer interrelaciones con él (Marcadores Periféricos), conforme la tabla anterior. Los resultados están disponibles en la tabla 1:

Tabla 1. Resultados de las búsquedas (DeCS/MeSH)

\begin{tabular}{lr}
\hline DESCRIPTORES & RESULTADOS DE LAS BÚSQUEDAS \\
\hline "Saúde Mental" & 5144 \\
"Assistência Integral à Saúde" & 1843 \\
"Padrão de Cuidado" & 18 \\
"Serviços de Saúde" & 5962 \\
"Serviços de Saúde Mental" & 1543 \\
"Serviços Comunitários de Saúde Mental" & 234 \\
\hline TOTAL & $\mathbf{1 4 . 7 4 4}$ \\
\hline
\end{tabular}

Fuente: Elaboración propia.

Después de visualizar las entradas individualmente para cada descriptor, se realizó un cruce entre ellas (tabla 2), y a partir del resultado se construyó un archivo con 782 fuentes de informaciones.

Tabla 2. Resultados de las búsquedas por cruzamiento (DeCS/MeSH)

\begin{tabular}{lr}
\hline DESCRIPTORES & RESULTADOS DE LAS BÚSQUEDAS \\
\hline "Saúde Mental" AND "Serviços de Saúde" & 77 \\
"Saúde Mental" AND "Serviços de Saúde Mental" & 510 \\
"Saúde Mental" AND "Serviços Comunitários de Saúde Mental" & 73 \\
"Assistência Integral à Saúde" AND "Serviços de Saúde" & 96 \\
"Assistência Integral à Saúde" AND "Serviços de Saúde Mental" & 27 \\
\hline
\end{tabular}


Tabla 2. (cont.)

\begin{tabular}{lr}
\hline DESCRIPTORES & RESULTADOS DE LAS BÚSQUEDAS \\
\hline "Assistência Integral à Saúde" AND "Serviços Comunitários de Saúde Mental" & 1 \\
"Padrão de Cuidado" AND "Serviços de Saúde" & 1 \\
"Padrão de Cuidado" AND "Serviços de Saúde Mental" & 0 \\
"Padrão de Cuidado" AND "Serviços Comunitários de Saúde Mental" & 0 \\
\hline TOTAL & $\mathbf{7 8 5}$ \\
\hline
\end{tabular}

Fuente: Elaboración propia.

El sistema de búsqueda de la BVS/PSI genera un catálogo con las informaciones claves sobre el contenido buscado. A partir del catálogo, se han eliminado las experiencias fuera del territorio brasileño, que se encuentran en referencias de Libros, Proyectos, Manuales, Documentos, Revistas, Tesis, Disertaciones y Obras Monográficas y Congresos Científicos. También se eliminaron, los textos que presentaban temas fuera del campo de interés de esta investigación, tales como: psicopatología, drogas desde la perspectiva de la patologización del uso, discusiones sobre teorías de la personalidad y otros.

La construcción de la mirada, o los criterios de inclusión, siguió las pistas de los textos sobre desinstitucionalización, Redes de Atención, Clínica Antimanicomial, Servicios y Dispositivos para la implementación de la Reforma Psiquiátrica, así como textos que abordaban la historia de la Reforma Psiquiátrica en Brasil y las políticas públicas, totalizando así 45 artículos.

Desarrollamos dos conjuntos de datos. Uno con los 45 artículos sistematizados en una tabla que en sus líneas contiene los artículos y en sus columnas la siguiente información: título, autor, fuente, año, resumen y descriptores. El otro, elaborado después de leer estos artículos en su totalidad, compuesto de 150 citas directas extraídas de los textos. No existe una metodología única para la producción de este tipo de información, pero es un procedimiento con un objetivo: constituir un corpus analizable ${ }^{19}$, entendiendo por corpus cualquier conjunto de textos o imágenes en un soporte material. Un corpus puede componerse de transcripciones de enunciados orales, de anotaciones y registros, de reproducciones de elementos gráficos, y textos previamente escritos o documentos ya existentes.

Para los análisis trabajamos con las contribuciones de la psicología discursiva y del movimiento construccionista para comprender la complejidad y la polifonía de ese campo. La primera fase de análisis se realizó leyendo y releyendo las citas para localizar los repertorios lingüísticos ${ }^{\mathbf{2 0}}$ presentes en el corpus sobre los sentidos del cuidado en la perspectiva de la atención psicosocial.

Los repertorios se pueden considerar como elementos esenciales que los hablantes utilizan para construir versiones de las acciones, los procesos cognitivos y otros fenómenos. De esta manera, cualquier repertorio está constituido por una restringida gama de términos usados, sea un estilo o sea una gramática específica. Ellos derivan de una o más metáforas clave, y la presencia de un repertorio a menudo está señalada por ciertos usos de palabras con un sentido figurado en el discurso ${ }^{14}$.

Específicamente, trabajamos con el uso de los Mapas Dialógicos ${ }^{21}$ como estrategia de aproximación y visibilidad de los repertorios lingüísticos para explorar los temas específicos sobre lo que hablan los textos. Finalmente, el Mapa se constituyó en una tabla organizada en columnas y distribuido en: columnas conteniendo cada una las citas y cómo éstas hablan (los repertorios). A partir de ello, con 
repetidas lecturas, (a) situamos cada cita, sus declaraciones y posiciones; (b) buscamos reconocer cómo cada cita describe, posiciona, localiza, justifica y argumenta el concepto del cuidado de salud mental; (c) problematizamos estas referencias y los efectos que producen para la perspectiva de la atención psicosocial y de la psicología.

A partir de ello fue identificada una serie de repertorios diferentes para nuestro tema general, que hemos agrupado en tres categorías: La Política Pública y la Reforma Psiquiátrica en Brasil; Modelos de asistencia y las controversias en la gestión y organización del proceso de trabajo; y la Salud Colectiva.

\section{Resultados}

Visibilizar los sentidos y entender la polifonía del lenguaje y sus usos ayuda a entender sus direccionamientos y efectos en ciertos campos de acción ${ }^{17}$. Esto ayuda también a percibir cómo un tema circula en los diferentes espacios y permite conocer las diferentes posiciones defendidas y asumidas por las personas que lo discuten, o sea, el tema no es neutral. Las bases de datos en este sentido, son, necesariamente, políticas. Los sentidos del cuidado en la perspectiva de la atención psicosocial presentes en las publicaciones analizadas permitieron comprender la complejidad de los procesos dinámicos de definición de esta categoría en el campo de la salud pública y de la reforma psiquiátrica brasileña.

En la categoría de las políticas públicas los repertorios están relacionados con la definición y presentación del concepto de atención como un marco legal presentado por un conjunto de leyes, políticas, programas y estrategias para las políticas públicas de salud mental en Brasil y las relaciones con el contexto histórico de la reforma psiquiátrica brasileña. La categoría de los modelos de asistencia y las controversias en la gestión y organización del proceso de trabajo incluyen los repertorios que se refieren al cuidado como una experiencia en los procesos de trabajo, una cierta forma de operar los modelos de atención que implican: prescripción, terapia y tratamiento, y también como un marcador ético y político que se relaciona con la producción de tecnologías ligeras, tales como: vinculación, recepción, coproducción de cuidados, integralidad, equidad, educación y prácticas compartidas.

Finalmente, la categoría de la salud colectiva y los repertorios apuntan a conceptos y prácticas de producción del cuidado como el desarrollo de un campo de conocimiento que se opone al modelo reduccionista de señales-síntomas-procedimiento, organizándose desde la libertad, autonomía, participación y garantía de derechos.

Por lo tanto, los repertorios no deben ser entendidos como delimitados o definitivos, sino como formas de pensar los movimientos, las relaciones y los conceptos, que están siempre en procesos de intercambio, de transversalidad y de establecimiento de redes entre ellos ${ }^{22}$.

\section{Política pública y la Reforma Psiquiátrica en Brasil}

Los sentidos del cuidado aquí se refieren a las definiciones que se producen a partir del marco legal de las políticas públicas. Los autores utilizan las definiciones legales e institucionales para contextualizar la trayectoria histórica de la reforma psiquiátrica y el cambio de paradigmas. Estas publicaciones expresan el carácter antagónico del proceso de implementación de estas políticas, así como las contradicciones del funcionamiento de una red de salud que convive con dos modelos que operan simultáneamente: el manicomial y el antimanicomial:

Observamos que las prácticas relacionadas con la atención de los pacientes con trastornos mentales siguen manteniendo en gran parte las del manicomio, especialmente en lo que respecta a las prácticas de medicación ${ }^{27(579)}$. 
Sin embargo, desde una perspectiva anti-manicomial y con la creación de servicios sustitutivos para la implementación de la Raps, otros modelos pasan a formar parte de la vida cotidiana de los servicios. Los artículos presentan informes de investigación desarrollados en los diversos municipios brasileños que evalúan este proceso, basados en experiencias locales y políticas específicas relacionadas con la salud mental: Política Nacional de Humanización ${ }^{23}$, Política Nacional de Educación Permanente ${ }^{24}$, Política Nacional de Participación y Control Social 25 y Política Nacional de Prácticas Integrativas y Complementarias ${ }^{\mathbf{2 6}}$. Estas perspectivas apuntan a un sistema de salud que con el tiempo se ha ampliado, mejorado y se ha hecho complejo, y esto también: "aportó una nueva perspectiva a los sujetos que antes sólo tenían la hospitalización como experiencia asistencial"18(177).

Como la política engendra la implementación de otras lógicas de atención que implican la superación del modelo asilar, la atención psicosocial y la producción del cuidado aparecen como una necesidad se hace posible en el campo de las políticas públicas:

[...] modelo de gestión de la salud, en el que los gestores, trabajadores y usuarios, sea del sector salud o del ámbito intersectorial, puedan dialogar y deliberar sobre la conducción, implementación, financiamiento y evaluación de las políticas de salud pública, así como sobre los procesos de trabajo que se realizan en la rutina diaria de los servicios, produciendo así una gestión de los modos de atención, además del control de los datos, índices y hojas de cálculo, como se observa comúnmente6(805).

Otro repertorio que forma parte de esta categoría es la formación y cualificación de los trabajadores. El conocimiento incorporado por los profesionales y la forma en que experimentan el proceso de salud y enfermedad son puntos críticos del sistema de salud:
La formación de los trabajadores, sin embargo, es una de las áreas menos problematizadas en la formulación de las políticas de salud. Las discusiones sobre este tema se han venido desarrollando desde la concepción de un Sistema Único de Salud, y su implementación no estuvo necesariamente acompañada de la calificación de los profesionales para actuar en el camino de los principios del actual modelo de salud9(351).

En el ámbito de la salud mental la lucha es por el cuidado en libertad y se expresa en la Ley $n^{0} 10.216 / 2001^{7}$. Si bien esta legislación específica no hace referencia al concepto de cuidado (la palabra cuidado no aparece en el documento), siempre refiriéndose a modelos de asistencia y tratamiento, inaugura en el país el derecho y la protección de las personas con trastornos mentales, que se garantizan sin ninguna discriminación por motivos de raza, color, sexo, orientación sexual, religión, elección política, nacionalidad, edad, familia, recursos económicos y el grado de severidad o tiempo de evolución de su trastorno, o cualquier otro. Según Campos ${ }^{\mathbf{1 8}}$ :

La atención de la salud mental en Brasil en los
últimos 35 años ha sufrido transformaciones
de diversa naturaleza. Desde el punto inicial de
estos cambios, con la creación del Movimiento
de Trabajadores de Salud Mental en 1978, hasta
la aprobación de la Ley 10.216 en 2001 , y sus
cambios posteriores, se percibe un importante
conjunto de acciones que pretenden cambiar
la realidad de esta atención, impulsando el
proceso de desinstitucionalización a través de
la creación y ampliación de la red de atención
de salud mental18(175).

Entre 1978 y 2019, la sociedad brasileña se conformó de varias maneras, en la dirección del proceso de institucionalización, consolidación y avances democráticos, cuyo referencial fue la promulgación de la Constitución en 1988 ${ }^{10}$. La sociedad civil comenzó a tener una participación fundamental en la lucha por la garantía de los derechos, teniendo como hito 
el proceso de lucha por la redemocratización del país, que permitió un proceso de apertura lento y gradual, cuando nuevos personajes entran en escena ${ }^{27}$.

Lo que caracteriza a estos movimientos es la lucha diaria por la afirmación y extensión de los derechos, que se produce de manera localizada y fragmentada, expresando las necesidades de las minorías sociales, entre ellas las personas con trastornos mentales. Los movimientos políticos comienzan a ocupar la esfera pública, que incluso en el marco de una sociedad capitalista desigual marcada por el patrimonio extractivo colonial, empiezan a luchar por la salud, la educación, la vivienda y la libre circulación en la ciudad. Finalmente, se agregaron varios factores que tuvieron una repercusión favorable en los primeros años de implementación del SUS ${ }^{\mathbf{2 8}}$.

Entre los repertorios presentes en los artículos se encuentran los seguintes elementos: legislación; estructuración de la atención; garantía de acceso; eficiencia; políticas públicas; referencia y contrarreferencia; diagnósticos; tratamiento; profesionales inseguros; integralidad; atención a la crisis; planificación; reorganización de la atención; acciones para la expansión de la red; reforma psiquiátrica; desnaturalización; micropolítica y estrategias.

\section{Modelos de asistencia y las controversias en la gestión y organización del proceso de trabajo}

El cuidado como forma de organizar la gestión de los procesos de trabajo también se refiere a cómo el concepto ha inducido nuevas formas de producir atención sanitaria, o sea, van introduciendo contenido lingüístico específico para la organización de las explicaciones ${ }^{\mathbf{1 4}}$. Los artículos presentan una visión general de los diferentes modelos de atención de la salud mental, considerándolos como una:

Traducción para un proyecto de atención de la salud de principios éticos, legales, organizativos, clínicos, socioculturales y de lectura de la situación epidemiológica y un cierto diseño de aspiraciones sobre la vida sana29.

El cuidado se sitúa como una posibilidad inventiva en la vida cotidiana que pasa por el cambio en la forma de entender el proceso salud-enfermedad y las relaciones laborales:

[...] la invención de nuevas formas de producir salud implica necesariamente en la modificación del proceso de trabajo de los equipos, lo que implica la apropiación colectiva del reto de construir nuevas formas de rendición de cuentas ${ }^{16(315)}$.

El cuidado se presenta como un aspecto que involucra la dimensión de las prácticas de salud de los profesionales, a través de las cuales apuntan a transformar los flujos de trabajo y la resolutividad de la atención. Estas transformaciones están relacionadas con la implementación de políticas, como se mencionó anteriormente, y con el funcionamiento de los servicios incorporados desde de la Raps:

El proceso de trabajo en salud, como eje estructurador de la producción de cuidados, se centra en el trabajo vivo, es decir, se desarrolla en acción, en el momento exacto del encuentro entre el trabajador y el usuario. Se observa que el atributo principal del trabajo vivo es la libertad, es decir, que en el momento exacto en que el trabajador se encuentra con el usuario, éste tiene el control de este proceso, toma decisiones basadas en la manera en que significa el usuario y la atención de la salud. [...] Así, las normas político-institucionales que regulan los servicios de salud son efectivas, siempre y cuando 'habiten' los propios trabajadores. De lo contrario, en la libertad de vivir el trabajo, logrará lo que considere pertinente, definido por su conocimiento y el mundo de significados que lo conectan con la idea general de la producción de cuidados ${ }^{16(315)}$.

Los artículos también hablan sobre la cronificación del proceso de trabajo. Esta cronicidad 
aparece a menudo en las discusiones de los autores como una crítica que atraviesa la reorientación del modelo de salud. Esta tensión entre las nuevas prácticas y los viejos hábitos ${ }^{30}$, se debe a la polaridad entre los modelos de atención hospitalaria y comunitaria “[...] lo que se percibe en la práctica es la constitución de otras formas de cronificación, institucionalización y segregación"13(1220).

Estas ambigüedades están presentes de diferentes maneras, ya que el cuidado es un concepto abierto a su propia metamorfosis, y marcan el proceso de transición entre la atención, históricamente ligada al modelo hospitalario, y el cuidado, que inaugura la desinstitucionalización y la atención comunitaria en el ámbito de la atención psicosocial. Los autores describen este proceso como:

[...] El concepto de asistencia sanitaria va más allá de la lógica específica y caso por caso de la intervención farmacoterapéutica sola. La atención se opera en una acción integral que incorpora tecnologías, significados y significados orientados a la comprensión de la salud

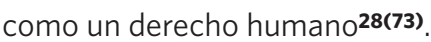

La intervención farmacoterapéutica hace referencia a las perspectivas ideológicas, técnicas y organizativas que estructuran el modelo psiquiátrico actual:

Además, observamos que los procesos de trabajo se producen a partir de un modelo de salud tradicional, en el que el sujeto es visto como partes desarticuladas; no se valora el vínculo entre el usuario y el equipo, ni la integridad de la atención, características fundamentales para la atención psicosocial y territorial. La fragmentación de los cuidados se debe, en gran medida, al aumento creciente de las especialidades y a la forma en que se organizan los procesos de trabajo. Al trabajar de esta manera, se ve comprometida la posibilidad de llevar a cabo una clínica extendida, entendida como aquella cuya mirada se dirige hacia el sujeto y toda su complejidad, a diferencia de la clínica centrada sólo en la enfermedad y sus síntomas 24(360).

El trabajo en salud es del tipo llamado 'vivo', es decir, en general, no trabaja en líneas de producción mecanizadas; requiere que los trabajadores y equipos multiprofesionales operen tanto con normas y protocolos como con la variación de procedimientos y comportamientos según el caso y el contexto. Los artículos presentan la necesidad de organizar el proceso de trabajo ordenado por modelos de gestión compartida como vías para la producción de cuidados en red ${ }^{31}$.

Los elementos presentes en los repertorios en esta dimensión son: modelos de atención; matriciamiento; prácticas profesionales; nuevos modelos; respuestas apropiadas; procesos de trabajo; proyecto terapéutico; habilidades y capacidades; modelos de gestión; gestión de nuevos modos de atención; redes; cuidados extendidos; transformación de las prácticas; renovación de las prácticas; cambio de la forma de tratar; cambio de la forma de cuidar; cambio de la forma de aprender y enseñar; postura activa; modelos de red de cuidados y modelos técnicoasistenciales.

\section{Salud coletiva}

Los repertorios utilizados para construir el cuidado lo sitúan como un paradigma que redimensiona la salud mental en el campo de la salud pública. Hacen consideraciones sobre el proceso saludenfermedad como resultado de complejos procesos sociales que exigen un enfoque interdisciplinario, transdisciplinario e intersectorial, con la resultante construcción de una diversidad de dispositivos territorializados de cuidado y atención ${ }^{32}$.

El cuidado se basa en la desinstitucionalización, la rehabilitación psicosocial y la reintegración social como marco orientador de su práctica asistencial. Elementos como autonomía, participación, reconocimiento de la diversidad y corresponsabilidad se mencionan como factores clave para la producción de 'nuevas prácticas' en salud mental: 
En las nuevas prácticas de Salud Mental, se argumenta que el desarrollo de Proyectos Terapéuticos requiere la formulación y constitución de un nuevo paradigma, en el que los problemas de salud se entienden como fenómenos complejos y multifacéticos. Además, las estrategias de atención deben entenderse como un proceso continuo e integrado de acciones orientadas a responder a las necesidades de

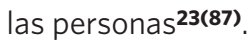

Es decir, el Proyecto Terapéutico sitúa las prácticas asistenciales como un dispositivo capaz de operar con tecnologías relacionales, colocando estos como modelos de atención que:

Supone ahora un enfoque más amplio, basado en la libertad, la integralidad, la confrontación de riesgos, la consideración de los derechos y la promoción de la posible singularidad y autonomía. Estos aspectos se entienden como una condición efectiva para el ejercicio de la inclusión y la ciudadanía, así como para emprender la tan deseada desinstitucionalización en el campo de la salud menta|10(916).

Las tecnologías pueden clasificarse como ligeras, ligera-duras y duras ${ }^{31}$. Todas ellas tratan la tecnología de forma integral, analizando todo el proceso de producción hasta el producto final. Las tecnologías ligeras son las de las relaciones; las tecnologías de ligera-dura son las de conocimiento estructurado, como las teorías, y las tecnologías duras son las de los recursos materiales.

Los sentidos de cuidado se refieren a su capacidad para efectuar cambios efectivos en la relación sujeto-objeto y cuestionar los modelos históricos de la salud pública brasileña. Para algunos autores ${ }^{33}$, la salud colectiva es una especie de metadiscurso supuestamente capaz de criticar y reconstruir conocimientos y procesos concretos de producción sanitaria:

Más que una política de resultados o de 'cura', es importante que los que llegan a estos servicios sean acogidos en primer lugar en sus singularidades y encuentren allí una referencia3(12)

El término salud colectiva ${ }^{34}$ comenzó a ser utilizado en Brasil en 1979, cuando un grupo de profesionales de la salud pública y de la medicina preventiva y social buscó fundar un campo científico con una orientación teórica, metodológica y política que privilegiaba lo social como categoría analítica:

la necesidad del desplazamiento esencial de la perspectiva de intervención de los hospitales psiquiátricos a la comunidad; el desplazamiento del centro de interés sólo de la enfermedad al sujeto en sufrimiento psíquico y a su discapacidad social y el desplazamiento de una acción individual a una acción colectiva en confrontaciones de los usuarios con sus contextos ${ }^{1(665)}$.

El cuidado también aparece como una manera de posicionarlo como una vía que marca el cambio de paradigma en el campo de la salud mental y la atención psicosocial:

Es necesario hacer uso de presupuestos clínicos cercanos a la vida concreta de las personas que acoge y a la producción de modos de vida que les son posibles. Para ello, es fundamental reunir a nuevos actores para activar otras formas de convivencia, de estar juntos y de vivir en sociedad ${ }^{8(469)}$.

De esta manera el cuidado debe alinearse de forma integral e intensiva, ofreciendo respuestas a las diversas dificultades que presentan los/as usuarios/as a lo largo de su vida. La clínica, se entiende como una producción que permite transformar la situación de las personas cronificadas por su larga estancia en instituciones disciplinarias ${ }^{35}$.

En esta dimensión aparecen en los elementos: movimientos contraideológicos y contrahegemónicos; repolitiza el sujeto y la salud; territorio; ciudadanía; superación del modelo asilar; premisas clínicas; clínica ampliada; reducción de daños; promoción; derechos; sociedad y emancipación. 


\section{Consideraciones finales}

Los artículos presentan una discusión sobre cómo el proceso de construcción de la política brasileña de salud mental produce de manera localizada el concepto de cuidado. Hacemos notar que el concepto se lo está escribiendo a medida que las experiencias se van construyendo en la vida cotidiana. En este sentido, el cuidado aparece como un concepto-experiencia que induce cambios paradigmáticos en el campo de las políticas públicas y la salud mental.

Considerando las estrategias metodológicas utilizadas en esta investigación, es posible comprender, a partir de la visibilidad de los repertorios lingüísticos, que, a nivel de las políticas públicas, de la organización y gestión de los procesos de trabajo, y de la salud colectiva, el cuidado se define como un concepto capaz de radicalizar las formas de pensar y producir la práctica de la atención psicosocial.

Los argumentos se ubican en la crítica al proceso de implementación de las políticas públicas, y en los avances y desafíos para una oferta de cuidados con tareas distintas: articular aspectos legales, conceptuales, históricos, culturales, económicos y sociales. De los textos se desprende que los servicios de salud mental, a menudo, no son capaces de organizar una oferta que permita al usuario transitar por las distintas redes que conforman la ciudad y, en este sentido, las prácticas terminan limitando la conducta prescriptiva y burocrática del cuidado.

Aunque se presentan como un concepto controvertido, los artículos, en general, critican la aplicabilidad del concepto cuando se reduce a las prácticas de cura y tratamiento. El corpus analizado permite comprender que este movimiento político del cuidado, como un concepto-experiencia, hace que la psicología también cuestione sus bases teóricas y epistemológicas, reposicionando el cuidado como una práctica transdisciplinaria que articula acciones y proyectos intersectoriales, actuando en el marco de la inseparabilidad entre lo clínico y lo político.

\section{Contribuyentes}

Santos RGA (0000-0003-0682-8880)* contribuyó sustancialmente al diseño, la planificación, el análisis y la interpretación de los datos; contribuyó de forma significativa a la redacción y revisión crítica del contenido. Íñiguez-Rueda L (0000-0002-1936-9428)* contribuyó sustan-cialmente al diseño, análisis e interpretación de los datos. 


\section{Referencias}

1. Costa-Rosa A, Luzio CA, Yasui S. Atenção psicossocial: rumo a um novo paradigma na saúde mental coletiva. In: Scliar M. Organizador. Archivos de saúde mental e atenção psi-cossocial. Rio de Janeiro: Nau Editora, 2003. p. 13-43.

2. Amarante P. Loucos pela vida - A trajetória da reforma psiquiátrica no Brasil. 2. ed. Rio de Janeiro: Fiocruz; 1995.

3. Damous I, Erlich H. O ambulatório de saúde mental na rede de atenção psicossocial: reflexões sobre a clínica e a expansão das políticas de atenção primária. Physis [internet]. 2017 [acceso en 2019 nov 17]; 27(4):911-932. Disponible en: http://www.scielo.br/scielo.php?script=sci_arttext $\&$ pid $=$ S0103$-73312017000400911 \& \operatorname{lng}=\mathrm{en}$

4. Bermudez KM, Siqueira-Batista R. "Um monte de buracos amarrados com barbantes": o conceito de rede para os profissionais da saúde mental. Saúde e Sociedade [internet]. 2017 [acceso en 2019 dec 17]; 26(4):904-919. Disponible en: https://doi.org/10.1590/ S0104-12902017170298.

5. Benevides R. Psicologia e o Sistema Único de Saúde: quais as interfaces? Rev. Psicol. Soc. [internet]. 2005 [acceso en 2019 abr 4]; (2):21-25. Disponible en: http://www.scielo.br/scielo.php?script=sci arttext\&pid=S0102-71822005000200004\&lng=en\& nrm=iso

6. Mattos RA. Os sentidos da integralidade: algumas reflexões acerca dos valores que merecem ser defendidos. In: Pinheiro R, Mattos RA, organizadores. Os sentidos da integralidade na atenção e no cuidado à saúde. Rio de Janeiro: IMS-UERJ; 2001. p. 39-64.

7. Brasil. Lei $\mathrm{n}^{0}$ 10.216, de 06 de abril de 2001. Lei da Reforma Psiquiátrica. Diário Oficial da União. 7 Abr 2001.

8. Brasil. Ministério da Saúde. Portaria n ${ }^{\circ} 3.088$, de 23 de Dezembro de 2011. Institui a Rede de Atenção Psicossocial para pessoas com sofrimento ou transtor- no mental e com necessidades decorrentes do uso de álcool, crack e outras drogas, no âmbito do Sistema Único de Saúde. Diário Oficial da União. 24 Dez 2011.

9. Campos GWS. Future prospects for the SUS. Ciênc. Saúde Colet. [internet]. 2018 [acceso en 2019 nov 23]; 23(6):1707-1714. Disponible en: https://doi. org/10.1590/1413-81232018236.05582018

10. Alves GTA, Santos RLC. A remanicomialização do cuidado em saúde mental no Brasil no período de 20102019: análise de uma conjuntura antirreformista. O Social em Questão [internet]. 2019 [acceso en 2019 oct 10]. Disponible en: http://osocialemquestao.ser. puc-rio.br/media/OSQ_44_art5.pdf.

11. Spink P. O pesquisador conversador no cotidiano. Psicologia \& Sociedade [internet]. 2008 [acceso en 2019 jun 20]; 20(esp):70-77. Disponible en: http://www. scielo.br/scielo.php?script=sci_arttext\&pid=S0102$-71822008000400010 \& \operatorname{lng}=e n \& n r m=$ iso.

12. Spink MJ, Frezza RM. Práticas discursivas e produção de sentidos: a perspectiva da Psicologia Social. In: Spink MJ. Práticas discursivas e produção do sentido no cotidiano - aproximações teóricas e metodológicas. São Paulo: Cortez; 2004. p. 27.

13. Serrano FJT, Argemí MD. Asociaciones heterogéneas y actantes: El giro postsocial de la teoría del actor-red. Rev. de Antropología Iberoamericana [internet]. 2005 [acceso en 2019 mayo 8]; (esp):01-26. Disponible en: https://dialnet.unirioja.es/servlet/ articulo? codigo $=1382237$.

14. Potter J, Wetherell M. El Análisis del Discurso y la Identificación de los Repertorios Interpretativos. In: Gordo AJ, Linaza JL. Psicologías, Discursos y Poder (PDP). España: Vi-sor Distribuciones; 1996.

15. Íñiguez RL. Investigación y evaluación cualitativa: bases teóricas y conceptuales. Aten-ción Primaria [internet]. 1999 [acceso en 2019 jun 27]; 23(8). Disponible en: http://www.unidaddocentemfyclaspal- 
mas.org.es/resources/1+Aten+Primaria+1999.+IC+ Bases + Teoricas $+y+$ Conceptos.pdf.

16. Lima TC, Mioto RCT. Procedimentos metodológicos na construção do conhecimento científico: a pesquisa bibliográfica. Rev. katálysis [internet]. 2007 [acceso en 2019 ene 19]; 10(esp):37-45. Disponible en: http://www.scielo.br/scielo.php?script=sci arttext\&pid=S1414-49802007000300004\&lng=en $\&$ nrm=iso.

17. Ribeiro MAT, Martins MHM, Lima JM. A pesquisa em base de dados: como fazer? In: Lang CE, Organizador. Metodologias: pesquisa em saúde, clínica e práticas psicológicas. Maceió: Edufal; 2015. p. 69-99.

18. Campos GWS. Saúde pública e saúde coletiva: campo e núcleo de saberes e práticas. Ciênc. Saúde Colet. [internet]. 2000 [acceso en 2019 nov 22]; 5(2):219-230. Disponible en: http://www.scielo.br/scielo.php?script=sci_arttext\&pid $=$ S1413$-81232000000200002 \& \operatorname{lng}=$ en.

19. Garay UA, Íñiguez RL, Martínez GM, et al. Evaluación cualitativa del sistema de reco-gida de sangre en Cataluña. Rev. Esp. Salud Publica [internet]. 2002 [acceso en 2019 jul 17]; 76(5):437-450. Disponible en: http://scielo.isciii.es/scielo.php?script=sci arttext\&pid=S1135-57272002000500006\&lng=es.

20. Aragaki SS, Piani PP, Spink MJ. Uso de repertórios linguísticos em pesquisas. In: Spink MJ. A produção de informação na pesquisa social: compartilhando ferramentas. Rio de Janeiro: Centro Edelstein de Pesquisas Sociais; 2014. p. 299-247.

21. Vanda LVN, Roberth MT, Pereira CCQ. O uso de mapas dialógicos como recurso analítico em pesquisas científicas. In: Spink MJ. A produção de informação na pesquisa social: compartilhando ferramentas. Rio de Janeiro: Centro Edelstein de Pesquisas Sociais; 2014. p. 247-273.

22. Amarante P, Torre EHG. Loucura e diversidade cultural: inovação e ruptura nas experiências de arte e cultura da Reforma Psiquiátrica e do campo da Saúde Mental no Brasil. Interface - Comun. Saúde Edu. [internet]. 2017 [acceso en 2019 sept 3]; 21(63):763774. Disponible en: https://doi.org/10.1590/180757622016.0881.

23. Brasil. Ministério da Saúde. HumanizaSUS: documento base para gestores e trabalhadores do SUS. 4. ed. Brasília, DF: MS; 2008.

24. Brasil. Ministério da Saúde. Portaria no ${ }^{0}$ 1.996, de 20 de agosto de 2007. Política Nacional de Educação Permanente. Diário Oficial da União. 21 Ago 2007.

25. Brasil. Ministério da Saúde. Portaria n ${ }^{0} 3.027$, de 26 de novembro de 2007. Aprova a Política Nacional de Gestão Estratégica e Participativa no SUS - ParticipaSUS. Diário Oficial da União. 27 Nov 2007.

26. Brasil. Ministério da Saúde, Secretaria de Atenção à Saúde, Departamento de Atenção Básica. Política nacional de práticas integrativas e complementares no SUS: atitude de ampliação de acesso. 2. ed. 1. reimpr. Brasília, DF : Ministério da Saúde; 2018.

27. Sader E. O anjo torto: esquerda (e direita) no Brasil. São Paulo: Brasiliense; 1995.

28. Amarante P, Torre EHG. Loucura e diversidade cultural: inovação e ruptura nas experiências de arte e cultura da Reforma Psiquiátrica e do campo da Saúde Mental no Brasil. Interface - Comun. Saúde, Edu. [internet]. 2017 [acceso en 2019 abr 11]; 21(63):763774. Disponible en: https://doi.org/10.1590/180757622016.0881 .

29. Campos GWS. Subjetividade e administração de pessoal: considerações sobre modos de gerenciar o trabalho em equipes de saúde. In: Merhy EE, Onoko R, organizadores. Agir em saúde: um desfio para o público. São Paulo: Hucitec; 1997. p. 229-66.

30. Ribeiro MC. Os Centros de Atenção Psicossocial como espaços promotores de vida: relatos da desinstitucionalização em Alagoas. Rev. Terapia Ocupac. [internet]. 2013 [acceso en 2019 jun 13]; 24(3):174-182. Disponible en: http://www.revistas.usp.br/rto/article/view/64900/pdf_24. 
31. Merhy EE. SAÚDE: A cartografia do trabalho vivo. 3. ed. São Paulo: Hucitec; 2002.

32. Yasui S, Costa-Rosa A. A Estratégia Atenção Psicossocial: desafio na prática dos novos dispositivos de Saúde Mental. Saúde debate [internet]. 2008 [acceso en 2019 mayo 21]; 32(78/79/80):27-37. Disponible en: https://www.redalyc.org/pdf/4063/406341773003. pdf.

33. Campos GWS. Saúde pública e saúde coletiva: campo e núcleo de saberes e práticas. Ciênc. Saúde Colet. [internet]. 2000 [acceso en 2019 nov 22]; 5(2):219-230. Disponible en: http://www.scielo.br/scielo.php?script=sci_arttext $\&$ pid $=$ S1413$-81232000000200002 \& \operatorname{lng}=$ en
34. Nunes ED. Saúde Coletiva: Revisitando a sua História e os Cursos de Pós-Graduação. Ciênc. Saúde Colet. [internet]. 1996 [acceso en 2019 mayo 17] ; 1(1):55-69. Disponible en: http://www.scielo.br/scielo.php?script=sci_arttext\&pid $=\mathrm{S} 1413-$ $-81231996000100055 \& \operatorname{lng}=$ en.

35. Lancetti A. Saúde mental nas entranhas da metrópole. In: Lancetti A, organizador. Saúde mental e saúde da família. 2. ed. São Paulo: Hucitec; 2001. p. 11-52.

Recibido el 31/12/2019

Aprobado el 13/08/2020

Conflicto de intereses: inexistente

Apoyo financiero: no hubo 